\title{
Spatial phase dislocations in femtosecond laser pulses
}

\author{
K. Bezuhanov, A. Dreischuh \\ Sofia University, Department of Quantum Electronics, \\ 5, J. Bourchier Blvd., BG-1164 Sofia, Bulgaria \\ G. G. Paulus ${ }^{1,2,3}$, M. G. Schätzel ${ }^{1}$, H. Walther ${ }^{1,2}$ \\ ${ }^{1}$ Max-Planck-Institut für Quantenoptik, \\ Hans-Kopfermann-Straße 1, \\ D-85748 Garching, Germany \\ ${ }^{2}$ Ludwig-Maximillians-Universität, Sektion Physik, \\ Am Coulombwall 1, D-85747 Garching, Germany \\ and \\ ${ }^{3}$ Texas A\&M University, Department of Physics, \\ College Station, TX 77843-4242, USA \\ D. Neshev, W. Królikowski, Yu. Kivshar \\ Australian National University, \\ Research School of Physical Sciences and Engineering, \\ Canberra ACT 0200, Australia
}

(Dated: December 21, 2004)

\begin{abstract}
We show that spatial phase dislocations can be generated in femtosecond laser beams by computergenerated holograms provided they are build in a setup compensating for the introduced spatial dispersion of the broad spectrum. We present analytical results describing two possible arrangements - dispersionless $4 f$-setup and double-pass grating compressor. Experimental results on the generation of optical vortices in the output beam of a $20 \mathrm{fs}-\mathrm{Ti}$ :sapphire laser and proof-of-principle measurements with broadband-tunable cw Ti:sapphire laser confirm the theoretical predictions.
\end{abstract}

PACS numbers: 050.1970; 090.1760; 120.5060; 140.3300; 140.7090

\section{INTRODUCTION}

The presence of phase dislocations in the wavefront of a light beam determines both the phase and intensity structure around them. Since the phase becomes indeterminate at the singularity point, both the real and imaginary parts of the field amplitude (i.e. also the field intensity) are zero [1]. Each one-dimensional $\pi$ phase dislocation is coupled with a zero-intensity line (one-dimensional odd dark beam; 1D ODB). An isolated point singularity with a screw-type phase distribution is associated with an optical vortex $(\mathrm{OV})$. The characteristic helical phase profiles of OVs are described by $\exp (i m \theta)$ multipliers, where $\theta$ is the azimuthal coordinate and the integer number $m$ is their topological charge (TC). As shown [2], an $m$-fold charged OV beam carries an orbital angular momentum of $m \hbar$ per photon independent on the spin angular momentum (i.e. on the polarization state). Recently, free space transfer of information encoded as orbital angular momentum was demonstrated [3], where the inherent security of the data depended on topological rather than on mathematical encryption.

The understanding the linear spatio-temporal behavior of focused femtosecond beams with phase singularities is of both theoretical and experimental interest. Remark- able spectral changes take place in the neighborhood of phase singularities near the focus of a converging, spatially fully coherent polychromatic wave [4]. Broadband illumination leads to non-negligible chromatic effects in the vortex region at a slight uncompensated spectral dispersion $[5,6]$.

In self-defocusing media the nonlinearity is able to compensate for the dark beam diffraction and dark spatial solitons have been generated [7-9] in a variety of materials. In self-focusing media OV beams are unstable [10], except the cases of partial incoherence [11], and/or non-local nonlinear response [12]. Instability-induced breakup of optical vortices to a controllable number of bright spatial solitons has lead to the concepts of 'soliton molecules' [14] and 'soliton algebra' [15-19]. All types of soliton applications will benefit from ultrashort pulses carrying spatial phase dislocations since such pulses exhibit high peak intensity, enough to access optical nonlinearities in many materials. These concepts along with the problems to generate subpicosecond helical (spinning) solitons in optical fibers [20] and stable spinning optical solitons in three dimensions [21] indicate the importance of the problem. Phase dislocations in femtosecond laser fields may also provide a new degree of freedom in experiments such as phase-controlled high-harmonic 
generation.

The challenge in creating spatial phase dislocations in short pulses with broad spectral bandwidths is to impose the desired dislocation onto all spectral components while keeping the pulse width and shape undistorted [22] and the pulse front untilted. The known methods for generating phase singularities applicable in the $\mathrm{cw}$ and quasi-cw regime are not suited for femtosecond lasers. Astigmatic transverse mode converters $[23,24]$ can not be used directly since they require transverse modes higher than the fundamental $T E M_{00}$. The approach [25] to prepare a Hermite-Gaussian-like $\left(H G_{01}\right)$ mode at the entrance of the converter by splitting and spatially offsetting an $H G_{00}$ mode out of phase seems feasible but for femtosecond pulses it requires an additional interferometricallycontrolled delay line. Because of the emitted transverse mode in the femtosecond regime intracavity phase elements [26] and beam rotators [27, 28] are not applicable. Transparent spiral wave plates $[29,30]$ are less flexible in controlling dynamically the phase distribution as compared to a liquid-crystal modulators structured in pie slices $[31,32]$. They all preserve the beam path, the last one exhibits a high efficiency in energy conversion. In both cases, however, the magnitude of the phase jump of the dislocation will deviate from $\pi$ for the different spectral components of the short pulse and topological dispersion will be present [33]. Glass platelets of a varying thickness providing linear phase retardation on one half of a (cw) laser beam and are able to produce optical vortices [34]. Because of the space-dependent dispersion and time delays this technique can not be applied to ultrashort pulses.

A well known [35] and widely used method to generate spatial phase dislocations is the reconstruction of computer-generated holograms $(\mathrm{CGH})$. This method is used to demonstrate screw [36, 37], step [38, 39] and mixed type dislocations [40] as well as arrays of such dislocations [41] in first-order diffracted beams. In order to impose the encoded phase dislocation onto all spectral components of the ultrashort pulse while keeping the pulse undistorted, the CGH has to be aligned as a part of an optical system with compensated spatial dispersion. We demonstrated recently [42] that a dispersionless $4 f$ system [43-45] is one possible solution of the formulated problem. Here we extend the investigations and demonstrate results obtained with 20 -fs laser pulses. Even well suited for femtosecond oscillators, in schemes involving (chirped pulse) amplifiers problems with the restless behavior of the dislocations and with amplified spontaneous emission in their cores have to be expected. In the second part of this work we show that spatial phase dislocations can be encoded in amplified femtosecond laser pulses at the later stage of the pulse shortening when the $\mathrm{CGH}$ is build in a double-pass grating-pair compressor setup [46].

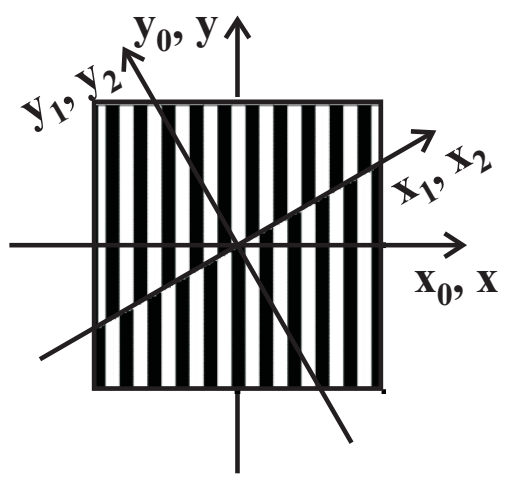

FIG. 1: CGH for generating an 1D ODB in a general (nonparallel) orientation of the dislocation axis with respect to the chosen coordinate system $\left(x_{0}, y_{0}\right)$.

\section{THEORETICAL MODEL}

Without loss of generality, in our analysis we normalize the electric field amplitude to unity and assume that the spatial profile of the optical field is Gaussian $E \sim \exp \left[-\left(x_{0}^{2}+y_{0}^{2}\right) / \sigma^{2}\right]$, where $\sigma$ is the beam width at $1 / e$-level and the aperture of the $\mathrm{CGH}$ is large enough not to cause edge diffraction. The field evolution after passing the CGH is analyzed by using the Fresnel integral

$E(x, y, z=s)=\frac{\exp (i k s)}{i \lambda s} \iint E\left(x_{0}, y_{0}, 0\right) \exp \left(\frac{i \pi r^{2}}{\lambda s}\right) d x_{0} d y_{0}$

and just behind it it has the form

$$
E\left(x_{0}, y_{0}, 0\right)=T\left(x_{0}, y_{0}\right) \exp \left[-\left(x_{0}^{2}+y_{0}^{2}\right) / \sigma^{2}\right] .
$$

In the above expressions $r^{2}=\left(x-x_{0}\right)^{2}+\left(y-y_{0}\right)^{2}, \lambda$ is a particular wavelength within the generated spectral bandwidth, $k=2 \pi / \lambda$, and $T\left(x_{0}, y_{0}\right)$ is the grating transmission function containing the phase profile $\varphi\left(x_{0}, y_{0}\right)$ of the desired dislocation. This function can be expanded in a Fourier-series in terms of field amplitudes $C_{n}$ of the different diffraction orders $n$

$$
T\left(x_{0}, y_{0}\right)=\sum_{n=-\infty}^{\infty} C_{n} \exp \left[i n 2 \pi\left(x_{0} / d\right)\right] \exp \left[\operatorname{in} \varphi\left(x_{0}, y_{0}\right)\right]
$$

where $d$ is the period of the diffraction grating imprinted on the CGH. In the particular case of a plane phase profile $\varphi\left(x_{0}, y_{0}\right)=\varphi_{0}=$ const, Eq. 3 describes the transmission of a diffraction grating with stripes perpendicular to the $O x_{0}$-axis (see Fig. 1). The quantity $d \varphi_{0} /(2 \pi)$ corresponds to the offset of the central transmitting stripe from the center of the coordinate system $O x_{0} y_{0}$. The coefficients $C_{n}$ depend on the particular profile of the stripes. For a binary CGH of perfectly transmitting and reflecting stripes of equal widths $C_{n}=\sin (n \pi / 2) /(n \pi)$ [47].

When a plane phase front is used at the stage of the CGH generation, independent from the type of the en- 
coded dislocation the curvature of the CGH stripes decreases away from the singularity and the stripes appear perpendicular to the coordinate axis $O x_{0}$. Since we are interested in the \pm 1 st diffracted order beams only, which reconstruct the encoded phase profile, we will analyze the electric field distribution at distances, at which the diffracted orders are well separated.

\section{CREATION OF PHASE DISLOCATIONS BY A SINGLE CGH}

The structure of the CGH for generating an 1D ODB and the coordinate system assigned to it are shown in Fig. 1. In the general situation of a non-parallel orientation of the dislocation axis with respect to one of the coordinate system axes the dislocation position is given by the straight-line equation $y=x \tan \alpha$. The encoded $\pi$-phase jump causes an offset of the stripes by half period on both sides of the dislocation. This CGH (Fig. 1) can be considered as composed from two offset identical halfgratings. Let us assume that the grating is illuminated by a laser beam aligned in a way that the $1 \mathrm{D}$ dislocation crosses its center. The CGH transmission function can be written in the form

$T_{ \pm}\left(x_{0}, y_{0}\right)=C_{1} \exp \left(i \frac{2 \pi x_{0}}{d}\right) \exp \left\{i\left[\varphi_{0}+\operatorname{sgn}\left(y_{0}-x_{0} \tan \alpha\right) \pi / 2\right]\right\}$

where the subscript " \pm " refers to the value of the $s g n$ function (i.e. to the upper or lower half of the grating). The field just behind the grating is therefore given by

$$
E_{ \pm}^{\prime}\left(x_{0}, y_{0}\right)=T_{ \pm}\left(x_{0}, y_{0}\right) \exp \left[-\left(x_{0}^{2}+y_{0}^{2}\right) / \sigma_{0}^{2}\right]
$$

It is more convenient to evaluate the diffraction integral Eq. (1) in a coordinate system $O x_{1}, y_{1}$ with $x_{1}$-axis parallel to the $1 \mathrm{D}$ dislocation. After rotating the coordinate system at an angle $\alpha$ Eq. 5 becomes

$$
\begin{aligned}
& E_{y_{1}>0}^{\prime}\left(x_{1}, y_{1}\right)=\exp \left[-\left(x_{1}^{2}+y_{1}^{2}\right) / \sigma_{0}^{2}\right] T_{y_{1}>0}\left(x_{1}, y_{1}\right) \\
& E_{y_{1}<0}^{\prime}\left(x_{1}, y_{1}\right)=\exp \left[-\left(x_{1}^{2}+y_{1}^{2}\right) / \sigma_{0}^{2}\right] T_{y_{1}<0}\left(x_{1}, y_{1}\right)
\end{aligned}
$$

Following the beam propagation, in a coordinate system $O x_{2} y_{2}$ parallel to $O x_{1} y_{1}$ but located at a distance $z=s$ apart of it, the electric field amplitude of the diffracted wave $E\left(x_{2}, y_{2}\right)$ is described by a sum of two integrals

$$
\begin{aligned}
& E\left(x_{2}, y_{2}\right)=\frac{\exp (i k s)}{i \lambda s}\left[\int_{-\infty}^{\infty} \int_{0}^{\infty} E_{y_{1}>0}^{\prime}\left(x_{1}, y_{1}\right) \exp \left(i \frac{\pi r_{21}^{2}}{\lambda s}\right)+\right. \\
& \left.\int_{-\infty}^{\infty} \int_{-\infty}^{0} E_{y_{1}<0}^{\prime}\left(x_{1}, y_{1}\right) \exp \left(i \frac{\pi r_{21}^{2}}{\lambda s}\right)\right] d x_{1} d y_{1},
\end{aligned}
$$

where $r_{21}^{2}=\left(x_{2}-x_{1}\right)^{2}+\left(y_{2}-y_{1}\right)^{2}$. By the substitution $y_{1} \rightarrow-y_{1}$, denoting $r_{i}^{2}=x_{i}^{2}+y_{i}^{2}, i=1,2$ and after some

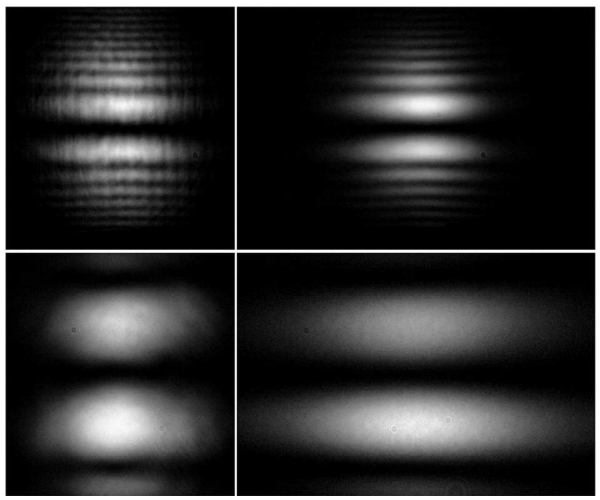

FIG. 2: Gray-scale images of 1D ODB $17 \mathrm{~cm}$ (top) and $35 \mathrm{~cm}$ (bottom) behind a single CGH, for cw (left) and fs laser beams (right).

routine mathematics the integrals can be unified and

$$
\begin{aligned}
& E\left(x_{2}, y_{2}\right)=\frac{2 C_{1}}{i \lambda s} \exp \left(i \varphi_{0}\right) \exp (i k s) \exp \left(i \frac{\pi r_{2}^{2}}{\lambda s}\right) \times \\
& \int_{-\infty}^{\infty} \int_{0}^{\infty} \exp \left(-\frac{r_{1}^{2}}{\sigma_{0}^{2}}\right) \exp \left(i \frac{\pi r_{1}^{2}}{\lambda s}\right) \times \\
& \exp \left[-i k x_{1}\left(x_{2}-\frac{\lambda s}{d} \cos \alpha\right)\right] \sin \left[\frac{k}{s} y_{1}\left(y_{2}+\frac{\lambda s}{d} \sin \alpha\right)\right] d x_{1} d y_{1}
\end{aligned}
$$

The equation describing the position of the dislocation $y_{2}+(\lambda s / d) \sin \alpha=0$ arises from the physical requirement for a zero value of the electric field amplitude $E\left(x_{2}, y_{2}\right)$ of the diffracted wave at the position of the phase dislocation. In a coordinate system $(x, y)$ with axes parallel to the initial $\left(x_{0}, y_{0}\right)$ axes (see Fig. 1$)$ the orientation of the dislocation at arbitrary $z=s$ is described by the condition

$$
y=(x-\lambda s / d) \tan \alpha .
$$

Hence, in the course of its propagation the dislocation remains parallel to this encoded in the $\mathrm{CGH}$, however there is a wavelength-dependent spatial offset (spatial dispersion) proportional to $\lambda s / d$. The only initial orientation of the 1D dislocation for which, for a broadband illumination of the $\mathrm{CGH}$, the integral intensity remains zero along the dislocation, is when it is encoded perpendicular to the CGH stripes (i.e. at an angle $\alpha=0$ ). This is intuitively easy to understand since the spatial dispersion is perpendicular to the CGH stripes. Unfortunately, spatial chirp is inevitable. Its presence can be clearly recognized in Fig. 2 by the horizontal elongation of the beam (along an axis, perpendicular to the grating stripes).

The gray-scale images of $1 \mathrm{D}$ ODB in $\mathrm{cw}$ and femtosecond regime are obtained by changing the operation regime of a Ti:sapphire Kerr-lens mode-locked oscillator. The oscillator being pumped by intracavity-doubled $N d: \mathrm{YVO}_{4}$ (Millenia Vi) laser emits nearly transformlimited 20-fs pulses at a repetition rate of $78 \mathrm{MHz}$ with an average power of $200 \mathrm{~mW}$ at a central wavelength of $797 \mathrm{~nm}$. In the measurements presented in the first part 


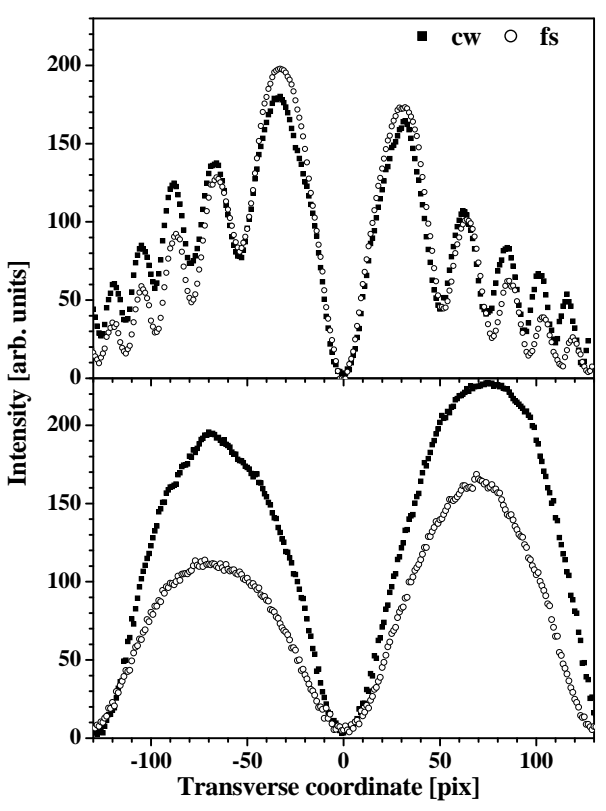

FIG. 3: Vertical cross-sections of the images shown in Fig. 2. Solid squares, cw regime; open circles, femtosecond regime.

of this work binary CGH produced photolithographically with a stripe period of $d=30 \mu \mathrm{m}$ are used and the experimental frames are recorded with a CCD-camera with $12 \mu \mathrm{m}$ resolution. In order to demonstrate the influence of the beam diffraction, in the left column of Fig. 2 frames of $1 \mathrm{D}$ ODBs in continuous-wave regime $17 \mathrm{~cm}$ and $35 \mathrm{~cm}$ behind the hologram are shown. The images in the right column are recorded at the same distances when modelocking was turned on. The beam broadening along the 1D dislocation due to the spatial dispersion is clearly much stronger pronounced in the femtosecond regime as compared to the cw one and it increases with increasing propagation path length. In Fig. 3 vertical cross-sections of the images shown in Fig. 2 are compared. As seen, in both regimes the integral intensity approaches the zerolevel at the phase dislocation encoded perpendicular to the grating stripes.

For an arbitrary orientation of the 1D dislocation the integral intensity can not be zero since the position of the dislocation in each monochromatic spectral component depends on $\lambda$. Pair of perpendicular 1D phase dislocations were encoded in another $\mathrm{CGH}$ at an dislocationto-stripe angle $\alpha=45 \mathrm{deg}$. The results observed in cwand femtosecond regime are shown in Fig. 4 for the same propagation distances $(17 \mathrm{~cm}$ and $35 \mathrm{~cm})$ after the $\mathrm{CGH}$. It is obvious that the $\mathrm{cw}$ quasi-2D dark beam retains its high contrast. Due to the spatial dispersion of the $\mathrm{CGH}$ the dislocations generated in the different spectral components in the femtosecond regime are displaced and the intensity modulation depth decreases along the propagation path. This results in the formation of horizontal grey stripes instead of a black cross.

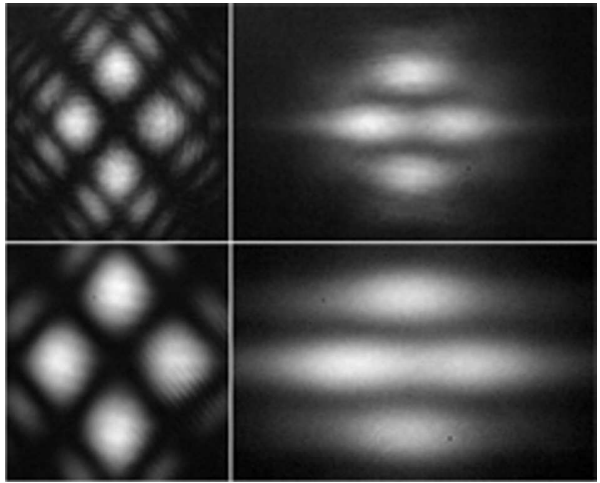

FIG. 4: Gray-scale images of a quasi-2D dark beam formed by crossed 1D phase dislocations, for $\mathrm{cw}$ and fs laser beams. Top, $17 \mathrm{~cm}$ behind a single CGH; bottom, $35 \mathrm{~cm}$ behind.

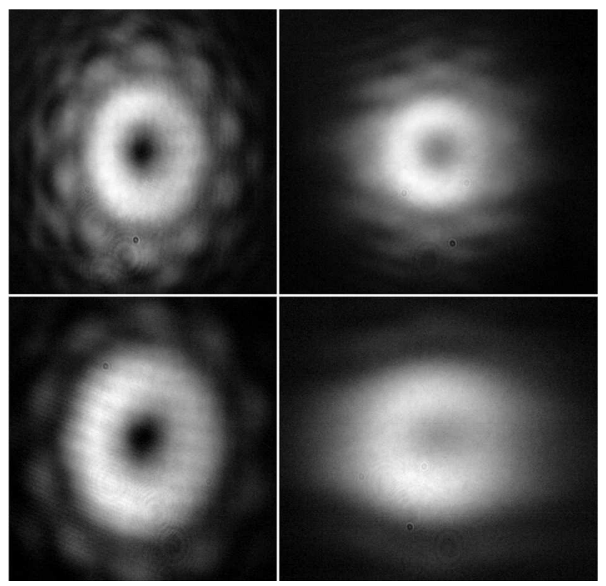

FIG. 5: Gray-scale images of OV beams $17 \mathrm{~cm}$ (top) and $35 \mathrm{~cm}$ (bottom) behind a single CGH, for $\mathrm{cw}$ and fs laser beams.

\section{TWO-DIMENSIONAL DISLOCATION AND ANALYSIS OF THE $4 f$-SYSTEM}

The optical vortex (OV) is a formation localized in two transverse dimensions. When it is generated by a single CGH the spatial dispersion displaces the vortices in the individual spectral components. This is clearly seen on Fig. 5, in which we show gray-scale images of OV beams recorded at two distances behind a $\mathrm{CGH}$, in both $\mathrm{cw}$ and fs regime. The contrast of the broadband formation is gradually reduced as compared to the contrast of the monochromatic OV beam under comparable conditions.

In the expansion of the grating transmission function $T\left(x_{0}, y_{0}\right)$ (Eq. 3 ) the multiplier accounting for the angular dispersion $\exp \left(i 2 \pi n x_{0} / d\right)$ does not depend on the particular form of the encoded phase profile $\varphi\left(x_{0}, y_{0}\right)$. This allows to compensate for the dispersion introduced by the CGH by using a suitable optical system involving an additional grating with the same period $d$ without any influence on the phase distribution $\varphi\left(x_{0}, y_{0}\right)$. This requirement is satisfied by a dispersionless $4 f$-system [43- 


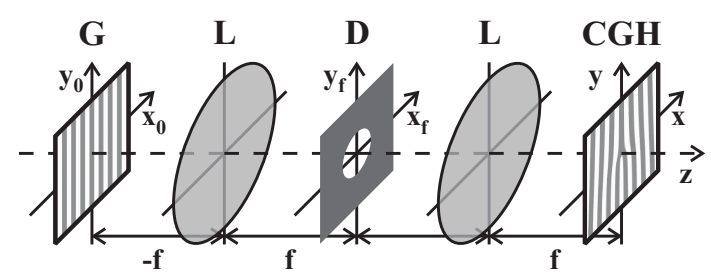

FIG. 6: Illustration of the $4 f$ setup that is analyzed theoretically. $G$, diffraction grating; $C G H$, computer-generated hologram with an encoded phase singularity; $L_{1}, L_{2}$, lenses of equal focal length $f ; D$, iris diaphragm. The input, Fourier, and output planes are denoted by indices $0, f$, and 1 , respectively.

\section{5] shown in Fig. 6.}

In order to obtain the evolution of the electric field inside the 4f-setup (Fig. 6) we use the integral relation between the field distributions in the front and back focal planes of a thin lens

$$
E\left(x_{f}, y_{f}\right)=\frac{1}{\lambda f} \iint E_{0} \exp \left[-i \frac{k}{f}\left(x_{0} x_{f}+y_{0} y_{f}\right)\right] d x_{0} d y_{0}
$$

which is obtained from the diffraction integral (Eq. 1) accounting for the transmission

$$
t(x, y)=\exp \left(i k n d_{0}\right) \exp \left[-i \frac{k}{2 f}\left(x^{2}+y^{2}\right)\right]
$$

of the thin lens of optical thickness $n d_{0}$ and focal length $f$. For simplicity, the constant phase multipliers and the quadratic phase terms introduced by the lenses are omitted. The lens apertures are considered to be much larger as compared to the spatial extent of the beam at the respective planes. In the particular case of an incoming Gaussian background beam, in the notations of the previous section, the first-order diffracted wave just after the first grating $G$ (see Fig. 6) is given by

$$
E^{\prime}\left(x_{0}, y_{0}\right)=C_{1} \exp \left(-\frac{x_{0}^{2}+y_{0}^{2}}{\sigma_{0}^{2}}\right) \exp \left(i \frac{2 \pi}{d} x_{0}\right)
$$

and its distribution $E\left(x_{f}, y_{f}\right)$ in the back focal plane of the first lens is

$$
E\left(x_{f}, y_{f}\right)=\frac{\sigma_{0}^{2}}{\pi \lambda f} C_{1} \exp \left[-\frac{\left(x_{f}-\frac{\lambda f}{d}\right)^{2}+y_{f}^{2}}{\left(\frac{\lambda f}{\pi \sigma_{0}}\right)^{2}}\right] .
$$

The analysis of 4f-type systems has been subject of extensive research in connection with their wide application in pulse shaping experiments [43-45]. In contrary, in this analysis the iris diaphragm does not affect the propagation of the first-order diffracted beam passing through the $4 f$-system and removes all other diffracted order beams only. Applying again the transformation Eq.(10) one gets the field distribution in front of the $\mathrm{CGH}$

$$
E(x, y)=\frac{C_{1}}{\lambda^{2} f^{2}} \exp \left[-\frac{x^{2}+y^{2}}{\left(\beta \sigma_{0}\right)^{2}}\right] \exp \left(i \frac{2 \pi}{\beta d} x\right),
$$

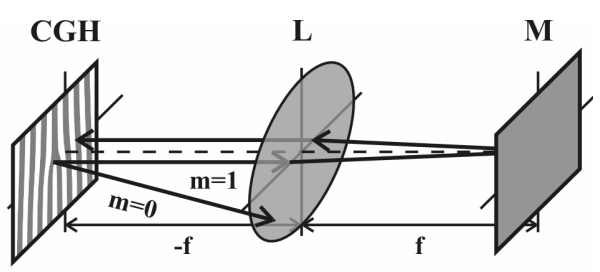

FIG. 7: Illustration of the folded $4 f$ setup that is used in the experiment. $C G H$, computer-generated hologram; $L$, quartz lens; $M$, silver-coated mirror.

where $\beta$ is the angular magnification of the optical system. The transmission function of the firstorder diffracted beam is given [47] by $T(x, y)=$ $A_{1} \exp (i 2 \pi x / d) \exp [i \varphi(x, y)]$. In this way we derived an analytical expression for the electric field amplitude $E^{\prime}(x, y)$ at the exit of the $4 f$-system:

$E^{\prime}(x, y)=\frac{C_{1} A_{1}}{(\pi \lambda f)^{2}} \exp \left[-\frac{x^{2}+y^{2}}{\left(\beta \sigma_{0}\right)^{2}}\right] \exp [i \varphi(x, y)] \exp \left[i \frac{2 \pi}{d}\left(1+\frac{1}{\beta}\right) x\right.$

The last multiplier in Eq. 15 accounts for the net spatial dispersion at the exit. For a perfect alignment $\beta=-1$ and the $4 f$-system is dispersion-free. Therefore, arbitrary oriented dark beams with phase dislocations generated in each individual spectral component are recombined spatially and temporally to overlap at the exit without spatial chirp.

The $4 f$-setup used in our experiment (Fig. 7) is folded in the Fourier plane by a silver-coated mirror. A largeaperture $(2.5 \mathrm{~cm})$ quartz lens with a focal length $f=$ $20 \mathrm{~cm}$ is aligned carefully to minimize aberrations. Binary $C G H$ of an optical vortex is positioned in a way to reconstruct the encoded point phase dislocation in the center of the background beam. In the peripheral part of this grating the stripes are parallel. This region is used as an effective second grating to recombine the spectral components at the exit. In Fig. 8a gray-scale images of optical vortices recorded $35 \mathrm{~cm}$ after the exit of the $4 f$ setup are shown. The frames are taken in $c w$ - and femtosecond regime successively by turning the mode-locking on/off and keeping the alignment unchanged. Interference lines in the frame recorded in $c w$ are clearly seen. They appear due to slight overlapping of the OV beam exiting the $4 f$-system with a beam reflected directly from the CHG substrate. Due to the lack of temporal overlapping and the reduction of the coherence length, both interference and speckles disappear in the $f s$ regime. Unlike Fig. 5, the contrast of the femtosecond OV is gradually improved and can be maximized by filtering out all parasitic reflections. An estimation based on the visibility of the interference structure in $c w$ regime (Fig. 8a, left frame) shows $(10 \pm 3) \%$ contribution of such reflections to the background signal in the vortex core. The influence of these reflections in fs-regime is likely to be stronger since the directly "reflected" broadband signal is actually dispersed in space. In Fig. 8b we show vertical (top) and horizontal (bottom) cross-section of the cw- and fem- 


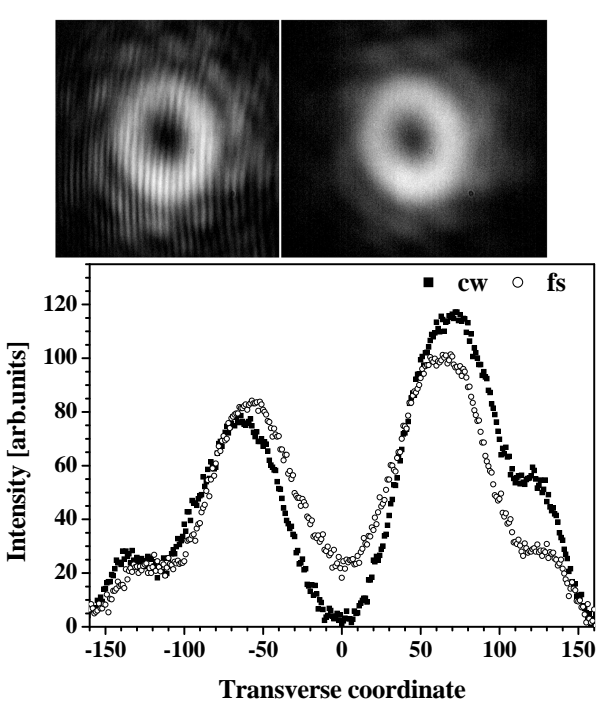

FIG. 8: Upper frames: Optical vortices recorded $35 \mathrm{~cm}$ after the $4 f$ setup in the $\mathrm{cw}$ and the femtosecond (fs) regimes. Graph: Corresponding vertical cross-sections of OV beams in cw and femtosecond regime (solid squares and open circles, respectively).

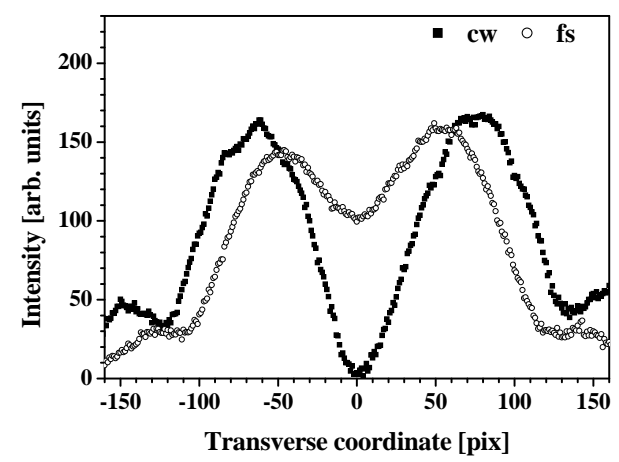

FIG. 9: Comparison between the OV cross sections taken parallel to the stripes of the CGH in the femtosecond regime. Open circles, single CGH; solid squares, folded $4 f$ setup.

tosecond OV beams (solid squares and open circles, respectively). The improvement of the contrast in the OV beam core is ones again confirmed (see Fig. 9) after comparing the beam profiles when a single $\mathrm{CGH}$ and a $\mathrm{CGH}$ in a $4 f$-system are used.

As far as no pulse shaping is performed in the Fourier plane, the low resolution $d \lambda / d x_{f}=150 \mathrm{~nm} / \mathrm{mm}$ related to the $30 \mu \mathrm{m}$ grating period is acceptable. Temporal pulse shaping in the same $4 f$-system would require much denser gratings and lenses/focusing mirrors of shorter focal lengths.

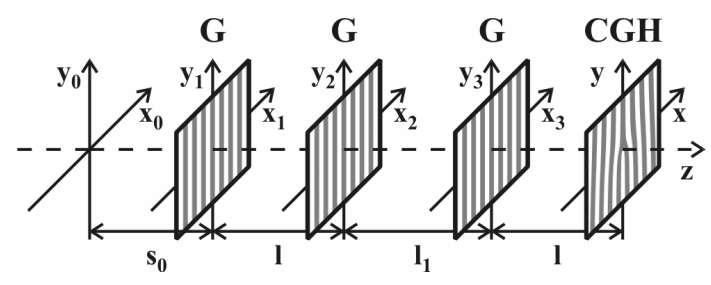

FIG. 10: Illustration of the double-pass grating compressor that is analyzed theoretically. $G$, diffraction gratings; $C G H$, computer-generated hologram with an encoded phase singularity; $l$, compressor length. The planes of the gratings are indexed successively. The CGH is assumed to stand at the exit of the compressor.

\section{CREATION OF PHASE DISLOCATIONS SIMULTANEOUSLY TO PULSE COMPRESSION}

In the following we will demonstrate that dark beams carrying phase dislocations can be generated in two-pass grating compressors [46] without introducing additional spatial dispersion. One of the gratings (in this theoretical model - the last one) has to be replaced by a CGH of a period $d$ equal to that of the other grating(s). The optical scheme analyzed is shown in Fig. 10.

Analytically, the procedure consists in evaluating the diffraction integral between the planes in which the diffraction gratings are located. In order to evaluate the field distribution in the plane $\left(x_{1}, y_{1}\right)$ of the first grating the input plane $\left(x_{0}, y_{0}\right)$ is shifted at a distance $z=s_{0}$ in front of it. (This shift is arbitrary and can be later set equal to zero.) Omitting the constant phase multipliers, the transmission functions of each grating and the $\mathrm{CGH}$ are described as follows

$$
\begin{aligned}
& T\left(x_{1}, y_{1}\right)=C_{1} \exp \left[i(2 \pi / d) x_{1}\right] \\
& T\left(x_{j}, y_{j}\right)=C_{1} \exp \left[-i(2 \pi / d) x_{j}\right], j=2,3 \\
& T(x, y)=C_{1} \exp [i(2 \pi / d) x] \exp [\varphi(x, y)] .
\end{aligned}
$$

Plus or minus sign in the phase corresponds to beam propagation in the first or in the minus first diffraction order of the respective grating. The electrical field distribution $E^{\prime}\left(x_{2}, y_{2}\right)$ just after the second grating can be obtained by multiplying the field diffracted between the $\left(x_{1}, y_{1}\right)$ and $\left(x_{2}, y_{2}\right)$ planes with the transmission function $T\left(x_{2}, y_{2}\right)$. The field distribution needed to evaluate the diffraction integral is a product of the field diffracted between the $\left(x_{0}, y_{0}\right)$ and $\left(x_{1}, y_{1}\right)$ planes and the transmission function $T\left(x_{1}, y_{1}\right)$ of the first grating. Therefore,

$$
\begin{aligned}
& E^{\prime}\left(x_{2}, y_{2}\right)=\frac{-C_{1}}{\lambda^{2} s_{0} l} \exp \left(-i \frac{2 \pi x_{2}}{d}\right) \exp \left[i k\left(s_{0}+l\right)\right] \times \\
& \iint\left[C_{1} \exp \left(i \frac{2 \pi x_{1}}{d}\right) \iint E\left(x_{0}, y_{0}\right) \exp \left(i \frac{\pi r_{10}^{2}}{\lambda s_{0}}\right) d x_{0} d y_{0}\right] \exp \left(i \frac{\pi r_{21}^{2}}{\lambda l}\right) d x
\end{aligned}
$$

where $r_{i j}^{2}=\left(x_{i}-x_{j}\right)^{2}+\left(y_{i}-y_{j}\right)^{2}, i, j=0,1,2$. Changing the order of integration and integrating over $x_{1}$ and $y_{1}$ the field after the first pass through the compressor (i.e. 
after the second grating; see Fig. 10) is

$$
\begin{aligned}
& E^{\prime}\left(x_{2}, y_{2}\right)=\frac{C_{1}^{2}}{i \lambda\left(s_{0}+l\right)} \exp \left[i k\left(s_{0}+l\right)\right] \exp \left[-i \frac{\pi s_{0} l \lambda}{\left(s_{0}+l\right) d^{2}}\right] \times \\
& \iint E\left(x_{0}, y_{0}\right) \exp \left[-i \frac{2 \pi l\left(x_{2}-x_{0}\right)}{\left(s_{0}+l\right) d}\right] \exp \left\{i \frac{\pi r_{20}^{2}}{\lambda\left(s_{0}+l\right)}\right\} d x_{0} d y_{0} .
\end{aligned}
$$

The evolution of the optical field amplitude during the second pass through the compressor [between the $\left(x_{2}, y_{2}\right)$ and the output $(x, y)$ plane] is modelled in the same way. Since the output grating of the compressor is chosen to be the CGH, the output electric field amplitude $E^{\prime}(x, y)$ contains the phase multiplier $\varphi(x, y)$. After some algebra

$$
\begin{aligned}
& E^{\prime}(x, y)=\frac{C_{1}^{2}}{i \lambda\left(l_{1}+l\right)} \exp \left[i k\left(l_{1}+l\right)\right] \exp [\varphi(x, y)] \exp \left[-i \frac{\pi l_{1} l \lambda}{\left(l_{1}+l\right) d^{2}}\right] \\
& \iint E^{\prime}\left(x_{2}, y_{2}\right) \exp \left[-i \frac{2 \pi l\left(x-x_{2}\right)}{d\left(l_{1}+l\right)}\right] \exp \left[i \frac{\pi r^{2}}{\lambda\left(l_{1}+l\right)}\right] d x_{2} d y_{2},
\end{aligned}
$$

where $r^{2}=\left(x-x_{2}\right)^{2}+\left(y-y_{2}\right)^{2}$. After substituting Eq. 18 in Eq. 19 and integrating over $x_{2}$ and $y_{2}$ the output electric field amplitude can be written in a compact form

$$
E(x, y)=C_{1}^{4} E_{d i f f} \exp \left\{i k\left[s-l(\lambda / d)^{2}\right]\right\} \exp [\varphi(x, y)]
$$

Here $E_{\text {diff } f}$ is the electric field amplitude diffracted in the course of the optical beam propagation in the compressor [accurate to accumulated linear phase $\exp (i k s)$; see Eq. 1]. The first phase term in Eq. 20 accounts for the different propagation path lengths (and transit times) of the different spectral components, i.e. for the negative group-velocity dispersion of the grating compressor. The last term contains the phase profile encoded in the CGH. Because of symmetry reasons the same result holds when the dislocation is generated by the first grating.

In the following, the behavior of the phase dislocations carried by broad bandwidth of femtosecond laser pulses is imitated by sets of measurements conducted with a cw laser tuned at different wavelengths. In that sense but without loss of generality, the following experiment is done as proof-of-principle one. The setup of the "grating compressor" is shown in Fig. 11. It consists of two identical phase masks (PM) of optical vortices. The PMs are phase CGHs fabricated directly on photoresist with stripe periods $80 \mu \mathrm{m}$. Their higher $(30 \%)$ efficiencies in first diffraction order and larger apertures $(1.2 \mathrm{~cm})$ were important in this measurement. In order to avoid dispersion in two spatial dimensions the gratings are pre-aligned under microscope on their holders to have parallel stripes. Then, the compressor scheme is aligned with the $532 \mathrm{~nm}$ output of a diode-pumped solid state laser (DPSSL, Verdi V5). Removing two mirrors (Fig. 11, dashed boxes) the same laser is used to pump a cw tunable Ti:sapphire ring laser (Coherent 899-21). The respective diffracted order beams after each PM are transmitted by two slits during the first pass through the "compressor". Plane silver-coated mirror is used to reflect the infrared beam back for the second pass. It intentionally introduces small vertical angular tilt (along

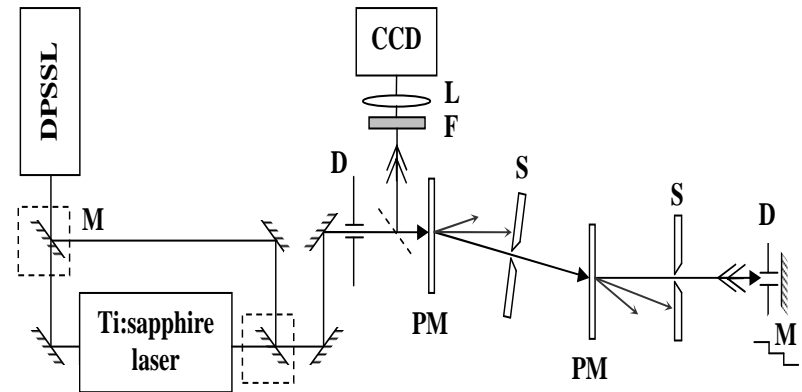

FIG. 11: Setup ot the proof-of-principle experiment. $P M$, phase masks (phase CGHs); $D$, iris diaphragm; $S$, slit; $M$, removable mirrors (dashed box) and folding mirror ensuring vertical offset in the reverse pass through the system; $F$, filter; ${ }_{L}$, imaging lens $(f=20 \mathrm{~cm}) ; D P S S L$, diode-pumped solid state laser (Verdi V5); Ti : Sa, cw tunable Ti:sapphire ring laser; $C C D$, charge-coupled device camera.

the grating stripes) which allows to separate the output beam from the input one and to encode a phase singularity only ones - at the entrance or at the exit of the setup even using identical phase CGHs. The output is imaged by a quartz lens $(f=20 \mathrm{~cm})$ directly on the array of a CCD-camera of $8 \mu \mathrm{m}$ resolution. Polarizing filter is used to avoid saturation of the recorded signal. The PM-toPM distances $(25 \mathrm{~cm}$ to $58 \mathrm{~cm})$ are chosen such that the individual diffraction orders can be separated. The PMto-folding mirror distance (limited by the half-aperture of the PMs $(1.2 \mathrm{~cm})$ ) was chosen in the same range.

The positions of the zero-th and \pm 1 -st order beams diffracted from the PM at the exit of the "compressor" are compared in Fig. 12 for different wavelengths. The particular emission wavelengths are measured with a wavemeter (Burleigh, WA-1100). Since in this measurement an $\mathrm{OV}$ is encoded in the beam by the first PM, it passes through the entire system and all three output beams carry OVs. This was done in order to use the OVs as spatial markers. Only one of the beams passes through the setup as it would be required a real double-pass grating compressor [46]. It is clearly seen that the OV nested on this beam preserves its position in space. The straight line in Fig. 12 represents a wavelength-independent OV position accurate within a standard deviation of 2 CCDcamera pixels when the laser wavelength is tuned in a $80 \mathrm{~nm}$ broad spectral interval. In contrast, the positions of the zero-order and "idler" first-order beams (middle and upper curve, respectively) change monotonically.

Since each ultrashort pulse is emitted having a broad spectrum and we measure the spatial positions of the vortices at discrete wavelengths, the experiment is a proofof-principle one. Nevertheless, taking the real spectrum of an amplified ultrashort pulse [48] and integrating a set of laser beam power density distributions recorded experimentally at discrete wavelengths, one can simulate the encoding of phase dislocations in a real double-pass grating compressor. The pairs of grayscale images inset in Fig. 13a,b are generated in this way. Fig. 13a refers 


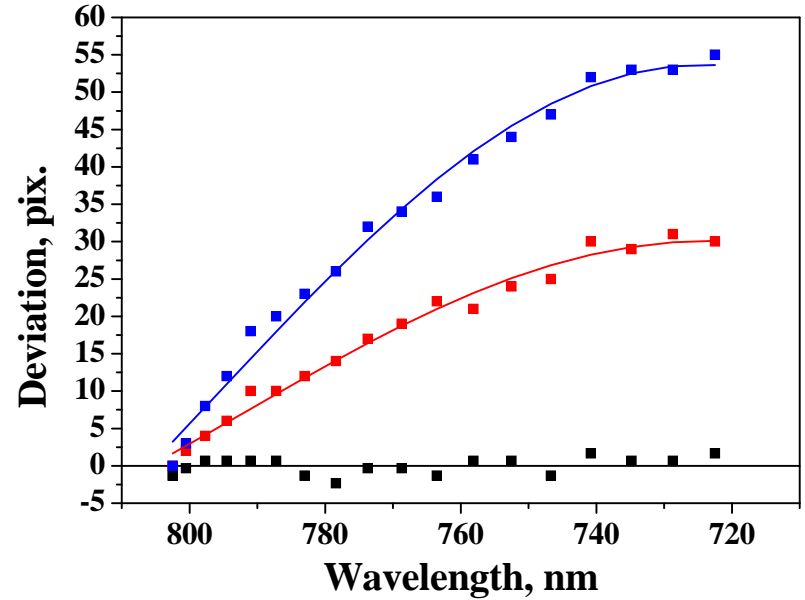

FIG. 12: Position of the OV at the exit for different wavelengths (bottom) and spatial deviation of the zero-order (middle) and idler OV beams when the dislocation is reproduced by the first phase mask.

to the $50 \mathrm{~cm}$ long "compressor", in which OV is encoded by the first PM. Because of the diffraction the spectrallyintegrated desired OV beam (right inset) is much broader as compared to this exiting the longer $(75 \mathrm{~cm})$ setup but encoded by the last PM (Fig. 13b, right inset). The uncompensated spatial dispersion of the different spectral components in the modelled "idler" beam (Fig. 13b, left inset) closely resembles the intensity distribution of an OV generated by a single CGH (Fig. 5, right column). The solid and dotted curves in Fig. 13a,b are diametral slices of the insets of the desired and "idler" output beams. The gradually higher contrast of the optical vortex generated in the "compressor" as compared to the contrast of the "idler" vortex in both cases strongly supports the general conclusion of this analysis: Dark beams carrying phase dislocations can be generated in chirped femtosecond laser beams at the stage of their pulse shortening in two-pass grating compressors.

\section{CONCLUSION}

Our analytical and experimental results demonstrate the possibility to create spatial phase dislocations in broadband (eg. femtosecond) optical fields by using CGHs. In order to cancel for the introduced spatial dispersion these suitably designed diffraction gratings have to be build in in $4 f$-setups or in double-pass grating com- pressors. The first approach does not affect the width of the ultrashort pulses and can be used directly with femtosecond oscillators. When (chirped pulse) amplifiers are involved in the femtosecond laser systems the phase dislocations can be generated in each spectral component at the later stage of the pulse shortening in a grating compressor. Our results are directly applicable to tunable laser beams when they have to preserve the positions of
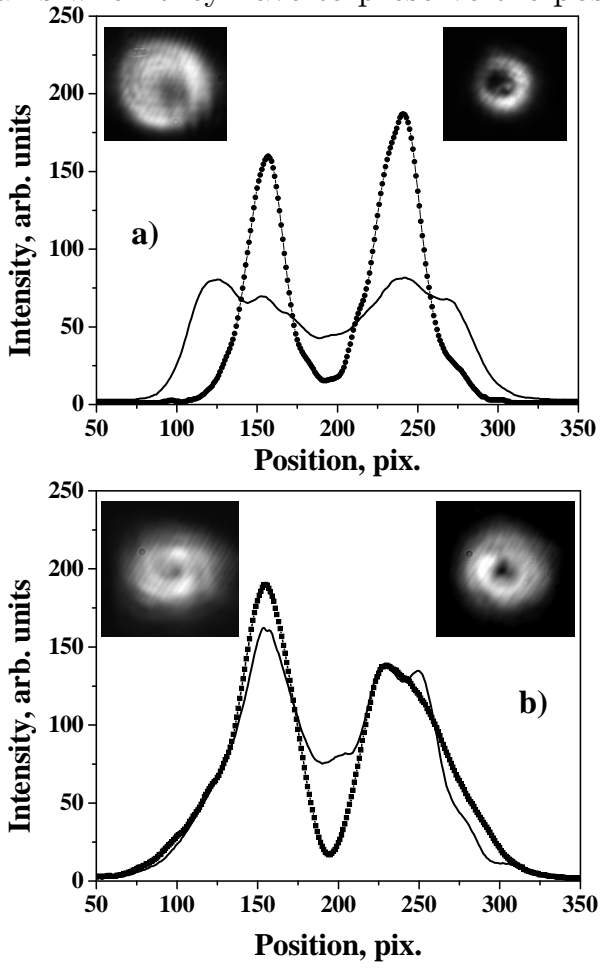

FIG. 13: Proof-of-principle simulations with OVs encoded in the first (a) and in the last (b) diffraction from a PM. Graphs: Transverse cross-sections of the idler (solid) and desired OV (dotted curve). Insets: Spectrally integrated experimental grayscale images (idler - left; desired OV - right).

the spatial phase dislocations, as well as to encode phase dislocations in white-light-type beams.

\section{Acknowledgments}

This work was partially supported by the National Science Fund (Bulgaria), under contract F-1303/2003, and the Australian Research Council.
[1] J. F. Nye, M. V. Berry, "Dislocations in wave trains," Proc. R. Soc. London A 336, 190-191 (1974).

[2] L. Allen, S. M. Barnett, M. J. Padget, Optical Angular Momentum (Institute of Physics, Bristol, 2004).

[3] G. Gibson, J. Courtial, M. J. Padgett, M. Vasnetsov, A.
Pas'ko, S. M. Barnett, S. Franke-Arnold, "Free-space information transfer using light beams carrying orbotal angular momentum," Optics Express 12, 5448-5456 (2004).

[4] G. Gbur, T. D. Visser, E. Wolf, "Anomalous behavior of spectra near phase singularities of focused waves," Phys. 
Rev. Lett. 88, 013901-1(4)(2002).

[5] M. V. Berry, "Coloured phase singularities," New Journal of Physics 4, 66.1 (2002).

[6] J. Leach, M. J. Padgett, "Observation of chromatic effects near a white-light vortex," New Journal of Physics 5, 154.1 (2003).

[7] G. A. Swartzlander, Jr., D. R. Andersen, J. J. Regan, H. Yin, A. E. Kaplan, "Spatial dark-soliton stripes and grids in self-defocusing materials," Phys. Rev. Lett. 66, 1583-1586 (1991).

[8] G. A. Swartzlander, Jr., C. T. Law, "Optical vortex solitons observed in Kerr nonlinear media," Phys. Rev. Lett. 69, 2503-2506 (1992).

[9] Yu. S. Kivshar, G. P. Agrawal, Optical Solitons, (Academic, San Diego, 2003).

[10] D. V. Skryabin, W. J. Firth, "Dynamics of self-trapped beamsm with phase dislocation in saturable Kerr and quadratic nonlinear media," Phys. Rev. E 58, 3916-3930 (1998).

[11] C.-C. Jeng, M.-F. Shih, K. Motzek, Yu. S. Kivshar, "Partially incoherent optical vortices in self-focusing nonlinear media," Phys. Rev. Lett. 92, 043904-1(4) (2004).

[12] A. I. Yakimenko, Y. A. Zaliznyak, Yu. S. Kivshar, "Stable vortex solitons in nonlocal self-focusing nonlinear media," http://lanl.arxiv.org/abs/nlin.PS/0411024.

[13] Wieslav "reference" http://lanl.arxiv.org/abs/nlin.PS/?.

[14] A. Desyatnikov, C. Denz, Yu. S. Kivshar, "Nonlinear optical beams carrying phase dislocations," J. Opt. A:Pure Appl. Opt. 6, S209-S212 (2004).

[15] D. V. Petrov, L. Torner, J. Martorell, R. Vilaseca, J. P. Torres, C. Cojocaru, "Observation of azimuthal modulational instability and formation of patterns of optical solitons in a quadratic nonlinear crystal," Opt. Lett. 23, 1444-1446 (1998).

[16] G. Molina-Terriza, J. Recolons, L. Torner, "The curious arithmetic of optical vortices," Opt. Lett. 25, 1135-1137 (2000)

[17] I. Freund, A. Belenkiy, "Higher-order extrema in twodimensional wave fields," J. Opt. Soc. Am. A 17, 434-446 (2000).

[18] L. Torner, A. P. Sukhorukov, "Quadratic solitons," Opt. Photonics News 13, 42-47 (2002).

[19] S. Minardi, G. Molina-Terriza, P. Di Trapani, J. P. Torres, L. Torner, "Soliton algebra by vortex-beam splitting," Opt. Lett. 26, 1004-1006 (2001).

[20] B. A. Malomed, G. D. Peng, P. L. Chu, "Helical versus fundamental solitons in optical fibers," Physica Scripta 63, 386-390 (2001).

[21] D. Mihalache, D. Mazilu, L.-C. Crasovan, I. Towers, A. V. Buryak, B. A. Malomed, L. Torner, J. P. Torres, F. Lederer, "Stable spinning optical solitons in three dimensions," Phys. Rev. Lett. 88, 073902-1(4) (2002).

[22] F. Grasbon, A. Dreischuh, F. Zacher, G.G. Paulus, H. Walther, "Femtosecond interferometric autocorrelations in the presence of pulse front distortions," Proc. SPIE 3571, 164-168 (1999).

[23] M. W. Beijersbergen, L. Allen, H. E. L. O. van der Veen, J. P. Woerdman, "Astigmatic laser mode converters and transfer of orbital angular momentum," Opt. Commun. 96, 123-132 (1993).

[24] M. J. Padgett, L. Allen, "Orbital angular momentum exchange in cylindrical-lens mode converters," J. Opt. B: Quantum Semiclass. Opt. 4, S17-S19 (2002).

[25] D. V. Petrov, F. Canal, L. Torner, "A simple method to generate optical beams with a screw phase dislocation," Opt. Commun. 143, 265-267 (1997).

[26] R. Oron, N. Davidson, A. Friesem, E. Hasman, "Efficient formation of pure helical laser beams," Opt. Commun. 182, 205-208 (2000).

[27] E. Abramochkin, N. Losevsky, V. Volostnikov, "Generation of spiral-type laser beams," Opt. Commun. 141, 59-64 (1997).

[28] A. V. Smith, D. J. Armstrong, "Generation of vortex beams by an image-rotating optical parametric oscillator," Optics Express 11, 868-873 (2003).

[29] M. W. Beijersbergen, R. P. C. Coerwinkel, M. Kristensen, J. P. Woerdman, "Helical-wavefront laser beams produced with a spiral phaseplate," Opt. Commun. 112, 321-327 (1994).

[30] A. G. Peele, P. J. McMahon, D. Paterson, Ch. Q. Tran, A. P. Mancuso, K. A. Nugent, J. P. Hayes, E. Harvey, B. Lai, I. McNulty, "Observation of an x-ray vortex," Opt. Lett. 27, 1752-1754 (2002).

[31] D. Ganic, X. Gan, M. Gu, M. Hain, S. Somalingam, S. Stankovic, T. Tschudi, "Generation of doughnut laser beams by use of a liquid-crystal cell with a conversion efficiency near 100\%," Opt. Lett. 27, 1351-1353 (2002).

[32] Q. Wang, X. W. Sun, P. Shum, "Generating doughnutshaped beams with large charge numbers by use of liquidcrystal spiral phase plates," Appl. Opt. 43, 2292-2297 (2004).

[33] G. A. Swartzlander, Jr., J. Schmit, "Temporal correlation vortices and topological dispersion," Phys. Rev. Lett. 93 093901-1(4) (2004).

[34] G.-H. Kim, J.-H. Jeon, K.-H. Ko, H.-J. Moon, J.-H. Lee, J.-S. Chang, "Optical vortices produced with a nonspiral phase plate," Appl. Opt. 36, 8614-8621 (1997).

[35] N. R. Heckenberg, R. McDuff, C. P. Smith, A. G. White, "Generation of optical phase singularities by computergenerated holograms," Opt. Lett. 17, 221-223 (1992).

[36] Z. S. Sacks, D. Rozas, G. A. Swartzlander, Jr., "Holographic formation of optical-vortex filaments," J. Opt. Soc. Am. B 15, 2226-2234 (1998).

[37] A. Dreischuh, G. G. Paulus, F. Zacher, F. Grasbon, H. Walther, "Generation of multiple-charged optical vortex solitons in a saturable nonlinear medium," Phys. Rev. E 60, 6111-6117 (1999).

[38] A. Dreischuh, G. G. Paulus, F. Zacher, "Quasi-twodimensional dark spatial solitons and generation of mixed phase dislocations," Appl. Phys. B 69, 107-111 (1999).

[39] A. Dreischuh, D. Neshev, G. G. Paulus, F. Grasbon, H. Walther, "Ring dark solitary waves: Experiment versus theory," Phys. Rev. E 66, 066611-1(7) (2002).

[40] A. Dreischuh, D. Neshev, G. G. Paulus, H. Walther, "Experimental generation of steering odd dark beams of finite length," J. Opt. Soc. Am. B 17, 2011-2017 (2000).

[41] A. Dreischuh, S. Chervenkov, D. Neshev, G. G. Paulus, H. Walther, "Generation of lattice structures of optical vortices," J. Opt. Soc. Am. B 19, 550-556 (2002).

[42] K. Bezuhanov, A. Dreischuh, G. G. Paulus, M. G. Schätzel, H. Walther, "Vortices in femtosecond laser fields," Opt. lett. 29, 1942-1944 (2004).

[43] A. M. Weiner, J. P. Heritage, E. M. Kirschner, "Highresolution femtosecond pulse shaping," J. Opt. Soc. Am. B 5, 1563-1572 (1988).

[44] M. B. Danailov, I. P. Christov, "Time-space shaping of light pulses by Fourier optical processing," J. Mod. Opt. 36, 725-731 (1989). 
[45] A. M. Weiner, "Femtosecond optical pulse shaping and processing," Prog. Quant. Electr. 19, 161-237 (1995).

[46] E. B. Treacy, "Compression of picosecond light pulses," Phys. Lett. A 28 34-35 (1968); id., "Optical pulse compression with diffraction gratings," IEEE J. Quantum Electron. QE-5, 454-458 (1969).

[47] W.-H. Lee, "Computer generated holograms: Techniques and applications" in Progress in Optics XVI, E. Wolf, ed. (Elsevier North-Holland, Amsterdam, 1978).

[48] F. Lindner, G. G. Paulus, F. Grasbon, A. Dreischuh, H. Walther, "Dispersion control in a 100-kHz-repetition-rate 30-fs Ti:sapphire regenerative amplifier system," IEEE J. Quant. Electron., QE-38, 1465-1470 (2002). 